\title{
PHARMACOTHERAPY OF NON-SMALL CELL LUNG CANCER: A SHORT REVIEW
}

\author{
BALAJI 0* \\ Department of Pharmacology, Kasturba Medical College, Manipal University, Manipal, Karnataka, India. Email:puntermmc@gmail.com \\ Received: 23 October 2017, Revised and Accepted: 01 December 2017

\begin{abstract}
Lung cancer is a global health problem with non-small cell lung cancer (NSCLC) being the most common histopathological variant causing almost $28 \%$ deaths in the United States of America. Platinum compounds were the mainstay of treatment, and since past 10 years, various newer targeted therapies have come into play. Epidermal growth factor receptor and anaplastic lymphoma kinase mutations play a major role in the development of advanced disease. Hence, targeted therapies and immunotherapies will remain an integral part in the management of advanced disease. Hence, this review focuses on the newer drugs approved by Food and Drug Administration to treat NSCLC.
\end{abstract}

Keywords: Tobacco, Programmed cell death pathway, Immune checkpoints, Platinum compounds.

(C) 2018 The Authors. Published by Innovare Academic Sciences Pvt Ltd. This is an open access article under the CC BY license (http://creativecommons. org/licenses/by/4. 0/) DOI: http://dx.doi.org/10.22159/ajpcr.2018.v11i3.23253

\section{INTRODUCTION}

Lung cancer incidence and mortality have been steadily increasing since 1930's due to the increase in cigarette smoking around the globe. Lung cancer has transformed from rare disease to a global health problem in the past 100 years. According to literature, lung cancer was identified among $50 \%$ miners working along the German border, and the disease was as called as bergkrankheit (mountain disease) in the early 1400 's. Later in the $18^{\text {th }}$ century, it was discovered that these miners had pulmonary sarcoma which was later in the $19^{\text {th }}$ century was confirmed as squamous cell carcinoma. It was also proposed the inhalation of dust, exposure to radiation and radon gas were some of the etiologies for developing lung cancer. Later in 1950 Antony Hill and his colleagues discovered cigarette smoking to be the main etiological factor in the development of lung cancer. In 1962 according to the royal college of physicians, a positive correlation was established between lung cancer and cigarette smoking and the research was published as a landmark article. At present around 80-85\% lung cancer deaths in the United States of America is directly attributable to smoking [1].

Lung cancer is histologically divided into two types with non-small cell lung cancer (NSCLC) being the predominant type (85\%) compared to small cell lung cancer (15\%). NSCLC is histologically divided into squamous cell type, adenocarcinoma and large cell tumors. Incidence of lung cancer was estimated to be around $12.7 \%$ of the total cancer incidence in the world with the United States of America having 28\% of cancer deaths due to lung cancer. It is the most common cancer affecting men and fourth most common cancer affecting women with age standardization rate of 33.8 per 10,0000 in males and 13.7 per 100,000 in females, respectively. In men and women, the lifetime probability of developing lung cancer was found to be 1 in 13 and 1 in 16 , respectively. In 2008, 1.38 million deaths were attributed to lung cancer with $43 \%$ death occurring due to lung cancer in developed countries compared to $57 \%$ in developing countries. The survival rates of lung cancer are found to be improving due to the recent development of newer treatment strategies as well as improved awareness among smokers with 5-year survival rates being $16.8 \%$ globally. It was also reported that women with smoking habit are more prone to development of lung cancer due to the difference in the carcinogenic effects of tobacco and also due to molecular aberrations which are more common and severe in women who smoke compared to men. Adenocarcinoma is said to be more common in women who smoke when compared to male smokers and adenocarcinoma is 4 times more common in non-smoking women compared to non-smoking males. This suggests a possible role of estrogen signaling is predisposing women to lung cancer [2-4].

Tobacco is the main risk factor in the development of lung cancer with $90 \%$ lung cancer deaths in the United States of America is mainly attributed to smoking. The polycyclic hydrocarbons present in the tobacco are responsible for p53 mutations leading to cell cycle dysregulation and carcinogenesis. Furthermore, $\mathrm{N}$ - nitrosamine compounds present in tobacco is said to have carcinogenic potential. There is a shift in the incidence of squamous cell type to adenocarcinoma due to reduced tar and increase in nitrosamines in cigarettes. According to the latest survey by U.S environmental protection agency radon exposure from soil is found to be a second most common risk after tobacco for development of lung cancer. Radon is carcinogenic due to the emission of $\alpha$ particle on decay. Asbestos, silica, beryllium, and chromium are other potential occupational toxins responsible for lung cancer. Other notable risk factors include human immunodeficiency virus, radiation exposure, and radiotherapy to chest wall for treatment of breast cancers [5].

Many mutations are known to be associated with lung cancer with most common ones being p53 mutation, epidermal growth factor receptor mutations, Kirsten rat sarcoma viral oncogene homolog (KRAS), and anaplastic lymphoma kinase $(A L K)$ mutations. Estimated glomerular filtration rate $(E G F R)$ mutations are more commonly associated with adenocarcinoma (30\%) compared to other histological types (2\%). It is also more commonly seen in never smokers and in women $(45 \%)$ compared to smokers (7\%). East Asian population is more frequently affected with EGFR mutations. KRAS gene encodes GTPase downstream EGFR. KRAS mutation results in activation of RAS signaling pathway with RAS proteins having impaired GTPase activity, leading to cell survival and cancer cell proliferation. KRAS mutations in comparison to EGFR are common in smokers. There is no sex predilection and whites are more frequently affected compared to Asians, with most predominant cancer type being adenocarcinoma. ALK encodes a receptor tyrosine kinase. ALK gene rearrangement is a mutation also implicated in the oncogenesis of NSCLC, especially adenocarcinoma [6-8]. With the development of various diagnostic tools and identification of molecular targets responsible for NSCLC, the treatment of this disease is on the upward trend. This has paved the way for the newer treatment options which include targeted therapy and immunotherapy, the main focus of this short review. 


\section{VARIOUS MODALITIES OF TREATMENT}

The treatment options include chemotherapy with platinum-based compounds, targeted therapies, immune therapies, and newer vaccines in development. Table 1 summarize the various drugs available for treatment.

\section{CHEMOTHERAPY}

\section{Platinum-based compounds}

Platinum-based compounds play a major role in the treatment of NSCLC. Cisplatin and carboplatin are two drugs that are widely used as a single agent and in combination with other anticancer drugs. Mechanism of action is mainly by covalently binding to purine deoxy ribonucleic acid (DNA) bases at N7 guanine and adenine. After substitution DNA cross-linking, occurs and DNA adducts are formed. These DNA adducts exert their anticancer activity through disruption of normal DNA signaling pathways by nucleotide substitution, chromosomal rearrangements, deletion, and also altering cell signaling pathways causing cell apoptosis. A meta-analysis of 51 randomized clinical trials showed platinum-based therapy improved 1-year survival rates to $10 \%$ and showed $27 \%$ reduction cancer deaths. A meta-analysis of 65 randomized control trials showed platinum compounds when used in combination with other agents as doublet chemotherapy improved survival rates from $30 \%$ to $35 \%$ and response rates from $13 \%$ to $26 \%$. Multicenter Italian Lung Cancer in the Elderly Study-2P showed recently the feasibility of using cisplatin-based combination therapies in elderly patients without any adverse outcomes. Furthermore, meta-analysis of 28 randomized trials comparing triple-drug regimens versus double drug platinum-based regimens showed that there is no improvement in survival rates but an improvement to be seen with response rates. The third-generation anticancer agents such as gemcitabine and vinorelbine are used widely in combination with platinum-based drugs currently days and have replaced the second-generation platinumbased combinations. When comparing cisplatin-based regimens versus carboplatin-based regimens, a recent meta-analysis comprising eight clinical trials revealed cisplatin when used in combination with newer agents had $11 \%$ more survival rate when compared to carboplatin used with newer agents [9].

The Lung Adjuvant Cisplatin Evaluation meta-analysis was conducted on the five largest, cisplatin-based studies (ALPI, BLT, IALT, JBR.10, and ANITA) and it clearly showed an improved response as well survival rates with cisplatin-based regimens. Major toxicities associated with platinum-based regimens include hematological toxicity, nephrotoxicity, and nausea/vomiting. Nephrotoxicity is more with cisplatin when compared to carboplatin. The Eastern Cooperative Oncology Group (ECOG) after a randomized trial on 8000 patients concluded addition of targeted therapy, namely, bevacizumab along with platinum-based regimens improved survival rates and reduced mortality by $20 \%$. However, addition of bevacizumab increased the incidence of adverse effects mainly pulmonary hemorrhage [9]. In spite of NSCLC having poorer prognosis and reduced survival rates, platinumbased compounds remain the backbone and mainstay of treatment of NSCLC. Addition of angiogenesis inhibitors with platinum compounds has clear-cut improvement in response and survival rates.

\section{Paclitaxel-based regimens}

Paclitaxel, the first member of the taxane group of anticancer drugs cause microtubule assembly and stabilizes tubulin polymer formation. This result in disruption of microtubule assembly needed for interphase and mitotic division. Paclitaxel, when used in combination with platinum compounds, showed improved survival and response rates. According to ECOG, cisplatin/paclitaxel combination was better when compared to cisplatin/etoposide combinations with overall survival rates in stage IIIB patients. Carboplatin in combination with paclitaxel showed less toxicity when compared to cisplatin/paclitaxel combination. The major dose-limiting toxicity was neuropathy with carboplatin-based regimens compared to severe nephrotoxicity with cisplatin/paclitaxel combination [10].

The carboplatin/paclitaxel regimen also reduces the myelosuppression seen when carboplatin was used as single agents due to the protective role of paclitaxel on platelets, by increasing the endogenous production of thrombopoietin and other cytokines, when used in dose $>75 \mathrm{mg} /$ $\mathrm{m}^{2}$. Docetaxel has demonstrated single-agent activity in advanced NSCLC as well as when used in combination with platinum compounds. $75 \mathrm{mg} / \mathrm{m}^{2}$ docetaxel was used in combination with platinum compounds in 3 weeks or 6 weeks regimens. There is no advantage of using docetaxel/cisplatin over docetaxel/carboplatin combination. Myelosuppression is the predominant toxicity observed with docetaxel in all these studies. Other unique toxicities include nail changes, hypersensitivity reactions, and pleural effusions [10].

\section{Gemcitabine based treatment}

Gemcitabine (2'deoxi-2', 2'-difluorocytidinemonohydrochloride) is a third-generation anticancer drug which is a novel deoxycytidine analog with structural similarity to cytosine arabinoside. Gemcitabine after getting inserted into the deoxycytidine side of DNA molecule, it results in addition of a neutral nucleoside molecule to the DNA strand. This protects gemcitabine from the DNA repair mechanisms trying to excise it. It also acts as a chain terminator. Gemcitabine used in a dose of $1250 \mathrm{mg} / \mathrm{m}^{2}$, on day 1 and day 8 every 3 weeks had improved response and survival rates on patients treated with other chemotherapy agents. When used as a single agent in both chemotherapeutically pre-treated and chemotherapy-naive patients, gemcitabine shows an objective tumor regression rate of approximately $20 \%$ [11].

Gemcitabine-based doublet therapy either in combination with platinum-based compounds or with non-platinum based compounds have shown clear-cut survival benefits and response rats in advanced disease. According to Spanish Lung Cancer Group which compared the outcomes with gemcitabine/platinum doublets and triplets to nonplatinum doublets, it was found the survival rates had no difference but the response rates were higher with gemcitabine/cisplatin doublet and triplet vinorelbine (42\% and 41\%) when compared to gemcitabine/vinorelbine $(27 \%)$ and gemcitabine/etoposide $(27 \%)$. Major toxicities associated with gemcitabine include nausea, vomiting, thrombocytopenia, asthenia, flu-like symptoms, and mild asthenia [11]. When compared to other anticancer drugs mild side effect profile of gemcitabine makes it is a good candidate for combination therapies.

Table 1: Various treatment modalities for NSCLC

\begin{tabular}{lllll}
\hline Chemotherapy & EGFR targeted therapy & ALK Targeted therapy & VEGF targeted therapy & Immunotherapy \\
\hline Cisplatin/Carboplatin & Erlotinib & Crizotinib & Bevacizumab & Nivolumab \\
Docetaxel & Gefitinib & Ceritinib & Ramucirumab & Pembrolizumab \\
Etoposide & Afatinib & Alectinib & & Atezolizumab \\
Gemcitabine & Osimertinib & & Ipilimumab \\
Paclitaxel & Cetuximab & & \\
Vinblastine & & & \\
Vinorelbine & & & \\
\hline
\end{tabular}

NSCLC: Non-small cell lung cancer, EGFR: Estimated glomerular filtration rate, ALK: Anaplastic lymphoma kinase, VEGF: Vascular endothelial growth factor 


\section{TARGETED THERAPIES}

\section{Vascular endothelial growth factor inhibitor}

Vascular endothelial growth factor is expressed by majority of NSCLC, and it is considered a marker for poor prognosis when overexpressed. Elevated circulating serum levels of vascular endothelial growth factor (VEGF) is found in almost all patients having NSCLC. VEGF is said to play an active role in the development of pleural effusions, lymph nodal metastasis and also in disseminated pleural malignancies. Bevacizumab was the monoclonal antibody targeted against VEGF in NSCLC. Even though it prolonged survival and response rates in NSCLC when combined with platinum-based compounds, there was an increased incidence of pulmonary hemorrhage in squamous cell patients. To overcome this liability, recently sequential chemotherapy with bevacizumab in combination with platinum-based compounds is in practice [12]. As per this regimen, cisplatin/carboplatin is given during first 2 cycles followed by cisplatin/carboplatin plus bevacizumab for up to 6 cycles and then bevacizumab alone till toxicity results. There was a significant reduction in the incidence of pulmonary hemorrhage when compared to previous regimens [12]

Recently ramucirumab was approved for NSCLC. Ramucirumab is a VEGF 2 antagonist, and it binds to VEGF-A, VEGF-B receptors. It is given as an infusion. Dose of ramucirumab for metastatic NSCLC is $10 \mathrm{mg} / \mathrm{kg}$, administered intravenously on day 1 of a 21-day cycle, before the infusion of docetaxel. REVEL trial showed patients who received ramucirumab plus docetaxel significant improvements in survival and response rates compared to the placebo plus docetaxel group. The response rate for ramucirumab plus docetaxel was $23 \%$ compared with $14 \%$ for placebo plus docetaxel group. Most common adverse effects noted include fatigue, neutropenia, diarrhea, epistaxis, stomatitis/ mucosal inflammation, and hypertension. Other agents in the trial include vademazan, sorafenib, sunitinib, pazopanib, axitinib, cediranib, and nintedanib [12].

\section{EGFR targeted therapy}

EGFR mutations as mentioned earlier are very common in never smokers and associated with the development of adenocarcinoma in women when compared to men. Gefitinib was the drug to be approved by Food and Drug Administration (FDA) in this class in 2003. It is a selective EGFR inhibitor. It acts by selectively attaching to ATP binding sites in the EGFR in patients with somatic EGFR mutations. T790 M mutation is responsible for resistance to gefitinib. It acts by blocking the growth of tumor cells, inhibiting receptor cell phosphorylation and also by preventing cell adhesion. It is used for Stage IV NSCLC with EGFR mutations or after failure with platinum-based therapies in advanced disease [13].

The next drug to be approved in this class was erlotinib by FDA in 2004. Erlotinib drug is used in Stage III or IV as a first-line treatment for NSCLC in patients where EGFR exon 19 deletions or exon 21 substitution mutations are present. Thus, it is used as a second- and third-line treatment in patients with EGFR wild-type NSCLC after failed chemotherapy and in combination with gemcitabine. On October 2016, FDA restricted the use of erlotinib for patients with specific EGFR mutations. The most recently approved ones include afatinib osimertinib and cetuximab. All act by the same mechanism of EGFR inhibition. Afatinib in addition also has HER-2 blocking activity and it is used in patients showing resistance to first-generation EGFR inhibitors. Osimertinib is a third-generation drug recently approved in 2015 and is mainly used in patients who have developed T790 M mutation due to treatment with other EGFR inhibitors. It acts by downregulating DNA synthesis and proliferation. Cetuximab and necitumumab are two monoclonal antibodies approved for treatment of advanced NSCLC. These antibodies are targeted against EGFR, and they attach outside the EGFR receptors when compared to other drugs which bind inside the EFGR domain. Both these drugs are given as infusion when compared to other EGFR inhibitors given as oral pills. Most common toxicity associated includes infusion reactions, diarrhea, vomiting, bleeding. Rashes, acne, bloating, liver abnormalities, mouth sores, and nail infections [13].

\section{ALK inhibitors}

Crizotinib is an oral small-molecule tyrosine kinase inhibitor targeting ALK, MET, and ROS1 tyrosine kinases. It was the first drug approved in 2013. It was approved after the PROFILE 1001 study which showed improved survival rates and response rats in advanced NSCLC. It is used as first-line agent in the treatment of Stage IV adenocarcinoma as a single agent. Notable side effects include vision disorder, diarrhea, nausea, vomiting, constipation, aminotransferase elevation, edema, upper respiratory infection, dysgeusia, and dizziness. Ceritinib is an ATP-competitive, tyrosine kinase inhibitor of ALK that inhibits the insulin-like growth factor 1 (IGF-1) receptor [31] and insulin receptor (INSR). It is used in patients in patients who have shown resistance to crizotinib. It is used as second-line agent in Stage IV advance adenocarcinoma resistant to Crizotinib. It is said to be more potent than crizotinib, diarrhea, vomiting, nausea, dehydration, ALT elevation, and hypophosphatemia. Alectinib, recently developed was designed to be a more selective and potent ALK inhibitor than crizotinib, in addition to activity against ALK, it has activity against the kinases LTK and GAK, but it is not active against INSR, IGF-1R, MET, or ROS1. It used as first-line or second-line treatment of patients with ALK-positive or metastatic NSCLC where crizotinib treatment failed. Dysgeusia, abnormal liver function tests, increased serum creatinine, rash, gastrointestinal side effects, decreased neutrophil count, increased serum creatine phosphokinase, stomatitis, and myalgias [14].

\section{Immunotherapy}

Immunotherapeutics is defined as treatment designed to elicit immune-mediated destruction of tumor cells. Checkpoint inhibitors targeting cytotoxic T-lymphocyte-associated antigen 4 (CTLA-4) and drugs targeting programmed death-1 (PD-1) pathway have been recently approved by FDA for treatment of advanced NSCLC. Monoclonal antibodies against CTLA-4 are designed to prevent the interaction between CTLA-4 and its ligands (CD80/CD86) resulting in blockade of the inhibitory signal provided by CTLA- 4 and subsequently enhancement of activation and proliferation of tumor-specific T-cells, thereby allowing an effective immune response against the tumor. Ipilimumab is one such monoclonal antibody. The PD- 1 is a T-cell surface receptor that is member of the B7-CD28, expressed on T cells, B cells, natural killer cells, activated monocytes, and dendritic cells. The role of PD-1 in normal human physiology is to limit autoimmunity by acting as a coinhibitory immune checkpoint expressed on the surface of $\mathrm{T}$ cells and other immune cells, including tumor-infiltrating lymphocytes. Nivolumab, pembrolizumab, and atezolizumab are recently FDA approved drugs as PD-1 inhibitor for the treatment of advanced NSCLC as second-line agent. Diarrhea, pneumonia, fatigue, and decreased appetite are some of the notable side effects with these agents [15].

\section{CONCLUSION}

In many cases of adenocarcinoma and squamous cell, NSCLC on the rise and various new mutations are identified, targeted therapies along with well-established anticancer drugs remain the mainstay of treatment in NSCLC. Various new drugs, as well as antibodies, are approved by FDA in the treatment of advanced NSCLC. Hence, hope the burden of NSCLC is reduced, and improvement in patient survival rates can be expected in the near future.

\section{REFERENCES}

1. Molina JR, Yang P, Cassivi SD, Schild SE, Adjei AA. Non-small cell lung cancer: Epidemiology, risk factors, treatment, and survivorship. Mayo Clin Proc 2008;83:584-94.

2. Youlden DR, Cramb SM, Baade PD. The international epidemiology of lung cancer: Geographical distribution and secular trends. J Thorac Oncol 2008;3:819-31.

3. Kamangar F, Dores GM, Anderson WF. Patterns of cancer incidence, mortality, and prevalence across five continents: Defining priorities to reduce cancer disparities in different geographic regions of the world. 
J Clin Oncol 2006;24:2137-50

4. Janssen-Heijnen ML, Coebergh JW. Trends in incidence and prognosis of the histological subtypes of lung cancer in North America, Australia, New Zealand and Europe. Lung Cancer 2001;31:123-37.

5. Risch HA, Howe GR, Jain M, Burch JD, Holowaty EJ, Miller AB. Are female smokers at higher risk for lung cancer than male smokers? A case-control analysis by histologic type. Am J Epidemiol 1993;138:281-93.

6. Shigematsu H, Gazdar AF. Somatic mutations of epidermal growth factor receptor signaling pathway in lung cancers. Int $\mathrm{J}$ Cancer 2006;118:257-62.

7. Pao W, Miller V, Zakowski M, Doherty J, Politi K, Sarkaria I, et al. EGF receptor gene mutations are common in lung cancers from "never smokers" and are associated with sensitivity of tumors to gefitinib and erlotinib. Proc Natl Acad Sci U S A 2004;101:13306-11.

8. Costa DB, Kobayashi S, Tenen DG, Huberman MS. Pooled analysis of the prospective trials of gefitinib monotherapy for EGFR-mutant non- small cell lung cancers. Lung Cancer 2007;58:95-103.

9. Tay K, Gutierrez M, Giaccone G. Platinum compounds in lung cancer: Current status. In: Platinum and Other Heavy Metal Compounds in Cancer Chemotherapy. New York: Humana Press; 2009. p. 231-42.

10. Belani CP. Paclitaxel and docetaxel combinations in non-small cell lung cancer. Chest 2000;117:144S-151S

11. Manegold C. Gemcitabine (Gemzar®) in non-small cell lung cancer. Expert Rev Anticancer Ther 2004;4:345-60

12. Piperdi B, Merla A, Perez-Soler R. Targeting angiogenesis in squamous non-small cell lung cancer. Drugs 2014;74:403-13.

13. Chan BA, Hughes BG. Targeted therapy for non-small cell lung cancer: Current standards and the promise of the future. Transl Lung Cancer Res 2015;4:36-54

14. Awad MM, Shaw AT. ALK inhibitors in non-small cell lung cancer: Crizotinib and beyond. Clin Adv Hematol Oncol 2014;12:429-39.

15. Massarelli E, Papadimitrakopoulou V, Welsh J, Tang C, Tsao AS. Immunotherapy in lung cancer. Transl Lung Cancer Res 2014;3:53. 